\title{
Continuum description of avalanches in granular media
}

\author{
Igor S. Aranson ${ }^{1}$ and Lev S. Tsimring ${ }^{2}$ \\ 1 Argonne National Laboratory, 9700 South Cass Avenue, Argonne, IL 60439 \\ 2 Institute for Nonlinear Science, University of California, San Diego, La Jolla, CA 92093-0402
}

(October 24, 2018)

\begin{abstract}
We develop a continuum description of partially fluidized granular flows. Our theory is based on the hydrodynamic equation for the flow coupled with the order parameter equation which describes the transition between flowing and static components of the granular system. This theory captures important phenomenology recently observed in experiments with granular flows on rough inclined planes (Daerr and Douady, Nature (London) 399, 241 (1999)): layer bistability, and transition from triangular avalanches propagating downhill at small inclination angles to balloon-shaped avalanches also propagating uphill for larger angles.
\end{abstract}

PACS: 45.70.-n, 45.70.Ht, 45.70.Qj, 83.70.Fn

Fundamental understanding of the dynamics of granular media still poses a challenge for physicists 113 and engineers [4]. The intrinsic dissipative nature of the interactions between the constituent macroscopic particles sets granular matter apart from conventional gases, liquids, or solids. One of the most interesting phenomena pertinent to the granular systems is the transition from a static equilibrium to a granular flow. The most spectacular manifestation of such a transition occurs during an avalanche. There has been a number of experimental studies of avalanche flows in large sandpiles [8,9] as well as in thin layers of grains on rough inclined surfaces 50 .

On the theoretical side, a significant progress had been achieved by large-scale molecular dynamics simulations 10,11 and by continuum theory 12 15. The current continuum approach to the description of avalanche flows in the physics community was pioneered by Bouchaud, Cates, Ravi Prakash and Edwards (BCRE) 13] and subsequently developed by de Gennes, Boutreux and and Raphaël 12, 14,15]. In their model is the granular system is spatially separated into two phases, static and rolling. The interaction between the phases is implemented through certain conversion rates. This model described certain features of thin near-surface granular flows including avalanches. However, due to its intrinsic assumptions, it only works when the granular material is well separated in a thin surface flow and an immobile bulk. In many practically important situations, this distinction between "liquid" and "solid" phases is more subtle and itself is controlled by the dynamics.

In this Letter we propose a new continuum model for multi-phase granular matter. The underlying idea of our approach is borrowed from the Landau theory of phase transitions [16]. We assume that the shear stresses in a partially fluidized granular matter are composed of two parts: the dynamic part proportional to the shear strain, and the strain-independent (or "static") part. The relative magnitude of the static shear stress is controlled by the order parameter $(\mathrm{OP})$ which varies from 0 in the "liquid" phase to 1 in the "solid" phase. Unlike ordinary matter, the phase transition in granular matter is controlled not by the temperature, but the dynamics stresses themselves. In particular, the Mohr-Coloumb yield failure condition [4] is equivalent to a critical melting temperature of a solid. The OP can be related to the local entropy [17] of the granular material. OP dynamics is then coupled to the hydrodynamic equation for the granular flow. We apply this model to study the transition to flow in thin granular layer on inclined planes with rough bottom. Our model captures important phenomenology observed by Pouliquen [7] and Daerr and Douady [5], including the structure of the stability diagram, triangular shape of downhill avalanches at small inclination angles and balloon shape of uphill avalanches for larger angles.

Model. The continuum description of the granular flow is based on the Navier-Stokes equation

$$
\rho_{0} D v_{i} / D t=\frac{\partial \sigma_{i j}}{\partial x_{j}}+\rho_{0} g_{i}, \quad j=1,2,3 .
$$

where $v_{i}$ are the components of velocity, $\rho_{0}=$ const is the density of material (we set $\rho_{0}=1$ ), $\mathbf{g}$ is acceleration of gravity, and $D / D t=\partial_{t}+v_{i} \partial_{x_{i}}$ denotes material derivative. Since the relative density fluctuations are small, the velocity obeys the incompressibility condition $\nabla \cdot \mathbf{v}=0$.

The central conjecture of our theory is that in partially fluidized flows, some of the grains are involved in plastic motion, while others maintain prolonged static contacts with their neighbors. Accordingly, we write the stress tensor as a sum of the hydrodynamic part proportional to the flow strain rate $e_{i j}$, and the strain-independent part, $\sigma_{i j}^{s}$, i.e. $\sigma_{i j}=e_{i j}+\sigma_{i j}^{s}$. We assume that the diagonal elements of the tensor $\sigma_{i i}^{s}$ coincide with the corresponding components of the "true" static stress tensor $\sigma_{i i}^{0}$ for the immobile grain configuration in the same geometry, and the shear stresses are reduced by the value of the order parameter $\rho$ characterizing the "phase state" of granular matter. Thus, we write the stress tensor in the form 


$$
\sigma_{i j}=\eta\left(\frac{\partial v_{i}}{\partial x_{j}}+\frac{\partial v_{j}}{\partial x_{i}}\right)+\sigma_{i j}^{0}\left(\rho+(1-\rho) \delta_{i j}\right) .
$$

Here $\eta$ is the viscosity coefficient. In a static state, $\rho=$ $1, \sigma_{i j}=\sigma_{i j}^{0}, v_{i}=0$, whereas in a fully fluidized state $\rho=0$, and the shear stresses are simply proportional to the strain rates as in ordinary fluids.

To close the system we need a set of constitutive relations between static shear and normal stresses, as well as an equation for the order parameter $\rho$. The issue of constitutive relations in granular materials is complex and not completely understood [4, 18. It appears that in many cases, the constitutive relations are determined by the construction history [19]. Recent studies indicated a fundamental role of the network of the force chains which carry forces longitudinally 20]. We will assume that for any given problem, the corresponding static constitutive relations have been specified.

For the order parameter $\rho$, we apply pure dissipative dynamics which can be derived from the "free-energy" type functional $\mathcal{F}$, i.e., $\dot{\rho}=-\delta \mathcal{F} / \delta \rho$. We adopt the standard Landau form for $\mathcal{F} \sim \int \boldsymbol{d} \mathbf{r}\left(D|\nabla \rho|^{2}+f(\rho, \phi)\right)$, which includes a "local potential energy" and the diffusive spatial coupling. The potential energy $f(\rho, \phi)$ should have extrema at $\rho=0$ and $\rho=1$ corresponding to uniform solid and liquid phases. According to the Mohr-Coulomb yield criterion for non-cohesive grains [4] or its generalization [20], the static equilibrium failure and transition to flow is controlled by the value of the non-dimensional ratio $\phi=\max \left|\sigma_{m n}^{0} / \sigma_{n n}^{0}\right|$, where the maximum is sought over all possible orthogonal directions $n$ and $m$ in the bulk of the granular material. We simply use this ratio as a parameter in the potential energy for the OP $\rho$. Without loss of generality, we write the equation for $\rho$ :

$$
\dot{\rho}=D \nabla^{2} \rho-a \rho(1-\rho) F(\rho, \phi)
$$

Further, according to observations we assume that the static equilibrium is unstable if $\phi \leq \phi_{1}$, where $\tan ^{-1} \phi_{1}$ is the internal friction angle for a particular granular material. Additionally, we assume that if $\phi<\phi_{0}$, the "dynamic" phase $\rho=0$, is unstable. Values of $\phi_{0}$ and $\phi_{1}$ do not coincide in general. Typically there is a range in which both static and dynamics phases co-exist (this is related to the so-called Bagnold hysteresis $[8]$ ). The simplest form of $F(\rho, \phi)$ which satisfies these constraints, is $F(\rho, \phi)=-\rho+\delta$, where $\delta=\left(\phi-\phi_{0}\right) /\left(\phi_{1}-\phi_{0}\right)$. Setting $D=1$ and $a=1$ we arrive at

$$
\dot{\rho}=\nabla^{2} \rho+\rho(1-\rho)(\rho-\delta) .
$$

For $\phi_{0}<\phi<\phi_{1}$ both static $(\rho=1)$ and dynamic $(\rho=0)$ phases are linearly stable, and Eq.(14) possesses a moving front solution which "connects" these phases. The speed of the front in the direction of $\rho=0$ is given by $V=$ $(1-2 \delta) / \sqrt{2}$. At $\delta=1 / 2$ both phases co-exist.

Chute flow. Let us now apply this formulation to a specific problem of the chute flow. We consider a layer of dry cohesionless grains on an inclined rough surface (see Fig.11). In the static equilibrium one has the following conditions:

$$
\sigma_{z z, z}^{0}+\sigma_{x z, x}^{0}=-g \cos \varphi, \sigma_{x z, z}^{0}+\sigma_{x x, x}^{0}=g \sin \varphi
$$

where the subscripts after commas mean partial derivatives. The solution to Eqs. (5) in the absence of lateral stresses $\sigma_{y y}^{0}=\sigma_{y x}^{0}=\sigma_{y z}^{0}=0$, is given by

$$
\sigma_{z z}^{0}=-g \cos \varphi z, \sigma_{x z}^{0}=g \sin \varphi z, \sigma_{x x, x}^{0}=0
$$

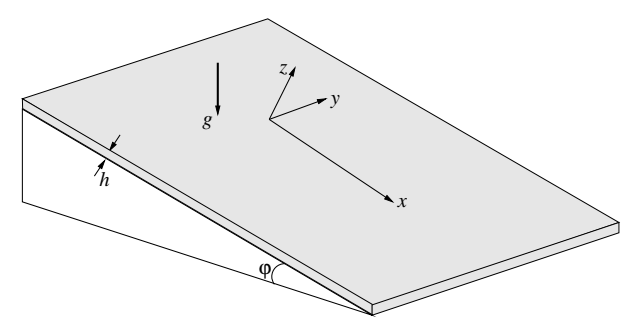

FIG. 1. Schematic representation of a chute geometry

In a static equilibrium there is a simple relation between shear and normal stresses, $\sigma_{x z}^{0}=-\tan \varphi \sigma_{z z}^{0}$. According to our conjecture, this relation between the static components of the stress is maintained in the flowing regime as well. For the chute flow geometry, the value of parameter $\phi$ in Eq. (4) can also be easily specified. In this case, the most "unstable" yield direction is parallel to the inclined plane, so we can simply write $\phi=\left|\sigma_{x z}^{0} / \sigma_{z z}^{0}\right|$.

Stationary solutions of Eq. (伍) for the confined chute geometry Fig. 1 are subject to the following boundary conditions (BC): no-flux condition $\rho_{z}=0$ at the free surface $z=0$, and $\rho=1$ at the bottom of the chute $z=-h$ (a granular medium is assumed to be in a solid phase near the rough surface). There always exists a stationary solution to Eq. (何 $\rho=1$ corresponding to a static equilibrium. For $\delta>1$ it is stable at small $h$, but loses stability at a certain threshold $h_{c}>1$. The most "dangerous" mode of instability satisfying the above boundary conditions, is $a \cos (\pi z / 2 h)$. The eigenvalue of this mode is $\lambda(h)=\delta-1-\pi^{2} / 4 h^{2}$, hence the neutral curve $\lambda=0$ for the linear stability of the solution $\rho=1$ is given by

$$
h_{c}=\frac{\pi}{2 \sqrt{\delta-1}} .
$$

For $h>h_{c}(\delta)$ grains spontaneously start to roll, and a granular flow ensues. In addition to the trivial state $\rho=1$, for $h>h_{s}(\delta)$ there exists a unique non-trivial stationary solution satisfying the above BC. The value of $h_{s}$ can be found as a minimum of the following integral as a function of $\rho_{0}$, the value of $\rho$ at the surface $z=0$,

$$
h_{s}=\min \int_{\rho_{0}}^{1} \frac{d \rho}{\sqrt{\frac{\rho^{4}}{2}-\frac{2(\delta+1) \rho^{3}}{3}+\delta \rho^{2}-c\left(\rho_{0}\right)}},
$$


where $c\left(\rho_{0}\right)=\rho_{0}^{4} / 2-2(\delta+1) \rho_{0}^{3} / 3+\delta \rho_{0}^{2}$. This integral can be calculated analytically for $\delta \rightarrow \infty$ and $\delta \rightarrow 1 / 2$. It is easy to show that for large $\delta$, the critical solution of Eq.(11) has a form $\rho=1+a \cos (k z)$ with $a \ll 1$ and $k=$ $(\delta-1)^{1 / 2}$, and therefore, $h_{s}(\delta) \rightarrow h_{c}(\delta)$. For $\delta \rightarrow 1 / 2$, the critical phase trajectory comes close to two saddle points $\rho=0$ and $\rho=1$, and an asymptotic evaluation of (8) gives $h_{s}=-\sqrt{2} \log (\delta-1 / 2)+$ const. This expression agrees with the empirical formula $\phi-\phi_{0} \sim \exp \left[-h_{s} / h_{0}\right]$ proposed in Ref. [5].

Neutral stability curve $h_{c}(\delta)$ and the critical line $h_{s}(\delta)$ limiting the region of existence of non-trivial granular flow solutions, are shown in Fig.2. They divide the parameter plane $(\delta, h)$ in three regions. At $h<h_{s}(\delta)$, the trivial static equilibrium $\rho=1$ is the only stationary solution of Eq. (14) for chosen BC. For $h_{s}(\delta)<h<h_{c}(\delta)$, there is a bistable regime, the static equilibrium state coexists with the stationary flow. For $h>h_{c}(\delta)$, the static regime is linearly unstable, and the only stable regime corresponds to the granular flow. This qualitative picture completely agrees with the recent experimental findings [5.7. Moreover, for rough bottom $\mathrm{BC}$ (corresponding to our $\rho=1$ ), authors of Ref. [5] found a region of bistability in the parameter plane $(h, \varphi)$ which has a shape very similar to our phase diagram Fig.2.

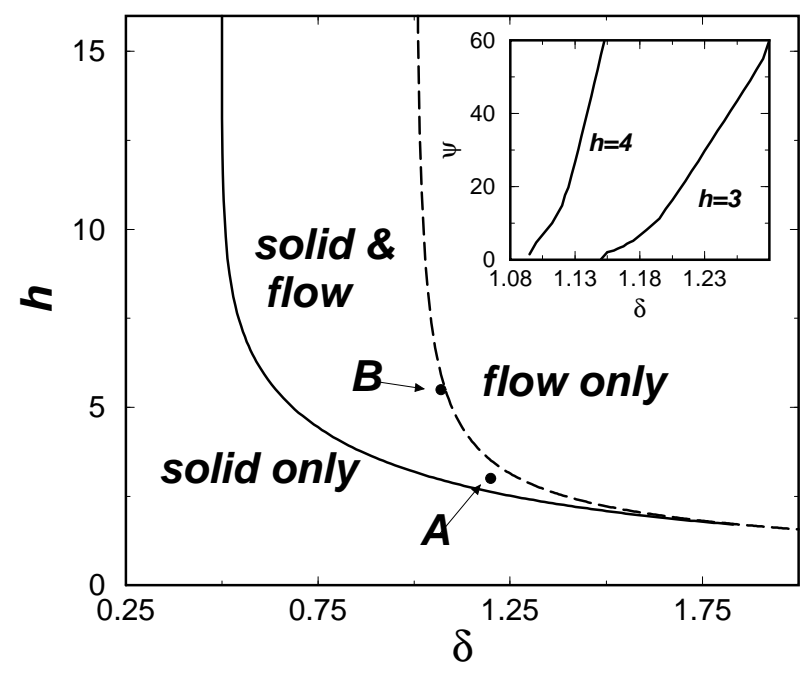

FIG. 2. Stability diagram. Dashed line shows the neutral curve $($ (7) , solid line shows the existence limit of fluidized state (8). Inset: $\psi$ (in degrees) vs $\delta$ for $\alpha=0.15$ and $\beta=0.25$

The velocity profile corresponding to a stationary profile of $\rho(z)$, can be easily found from Eq. (2),

$$
\eta \frac{\partial v_{x}}{\partial z}=g \sin \varphi z-\rho \sigma_{x z}^{0}=g \sin \varphi(1-\rho) z
$$

The flux of grains in the stationary flow $J$ is given by

$J=\int_{-h}^{0} v_{y}(z) d z=\frac{g \sin \varphi}{\eta} \int_{-h}^{0} \int_{-h}^{z}\left(1-\rho\left(z^{\prime}\right)\right) z^{\prime} d z^{\prime} d z$
Pouliquen [7] proposed a scaling for the mean velocity $\bar{v}=J / h$ vs thickness of the layer $h$ in the stationary flow regime, $\bar{v} \propto h^{3 / 2} / h_{s}$, which works for angles $\varphi$ as well as for different grain sizes. Eq. (10) yields $v \propto\left(h-h_{s}\right)^{1 / 2}$ for small $h-h_{s}$ and $v \propto h^{2}$ for large $h$. It is plausible that the experimentally found scaling exponent $3 / 2$ is the result of the crossover between two different regimes. However, renormalization $\bar{v} / \sqrt{g h}, h / h_{s}$ as in Ref. [7] does not collapse our results onto a single curve, perhaps due to the assumption of a simple Newtonian relation between the strain $v_{z}$ and the hydrodynamic part of the shear stress $\sigma_{x z}$ with a fixed viscosity $\eta$ (see Eq.(2)). In fact, $\eta$ itself may depends on $\rho$ and $z$ in some fashion.

For a deep chute $(h \gg 1)$, the stationary solution of Eq.(4) can be found analytically (cf. Ref. [21]). However, in this case the slope of the free surface may not be equal to the slope of the inclined plane, but is itself determined by the amount of sand which is poured on the surface upstream. Thus, the closure of the problem will be provided by the constraint $J=$ const.

Avalanches in shallow chute. In the vicinity of the neutral curve (7) Eqs.([1]:3) can be simplified. We look for solution in the form

$$
\rho=1-A \cos \left(\frac{\pi}{2 h} z\right)+\text { h.o.t., }
$$

where $A \ll 1$ is a slowly varying function of $t, x$, and $y$. Substituting ansatz (11) into Eq. (3) and applying orthogonality conditions, we obtain

$$
A_{t}=\lambda(h) A+\nabla_{\perp}^{2} A+\frac{8(2-\delta)}{3 \pi} A^{2}-\frac{3}{4} A^{3}
$$

where $\nabla_{\perp}^{2}=\partial_{x}^{2}+\partial_{y}^{2}, \lambda(h)=\delta-1-\frac{\pi^{2}}{4 h^{2}}$. Deriving this equations we assumed that $(2-\delta) A^{2}$ and $A^{3}$ are of the same order, i.e. $\delta \approx 2$, however qualitatively similar equation with a different nonlinearity can be obtained for any $\delta$ and $h$. Eq. (12) must be coupled to the mass conservation equations which reads as (here we neglect contribution from the flux along $y$-axis $\left.J_{y} \sim \partial_{y} h \ll J\right)$ :

$$
\frac{\partial h}{\partial t}=-\frac{\partial J}{\partial x}=-\alpha \frac{\partial h^{3} A}{\partial x},
$$

where $J$ was calculated from Eq. (10) and $\alpha=2\left(\pi^{2}-\right.$ 8) $g \sin \varphi / \eta \pi^{3}$. Taking into account that variations in $h$ also change local surface slope, we adopt $\delta=\delta_{0}-\beta h_{x}$ with $\beta=1 /\left(\phi_{1}-\phi_{0}\right)$.

We studied Eqs. (12,13) numerically. The simulations were performed in fairly large systems, 400 dimensionless units in $x$-direction (downhill), and 200 units in $y$ direction, with the number of grid points $1200 \times 600$ correspondingly. As initial conditions we used uniform static layer: $h=h_{0}, A=0$. We triggered avalanches by a localized perturbation introduced near the point $(y, z)=\left(L_{y} / 4, L_{z} / 2\right)$. Close to the solid line in Fig. (2) we indeed observed avalanches propagating only downhill, with the shape very similar to the experimental one. 
The avalanche leaves triangular trace with the opening angle $\psi$ in which the layer thickness $h$ is decreased with respect to original value $h_{0}$. At the front of the avalanche the layer depth is increased with respect to $h$, as in experiment. The opening angle as a function of $\delta$ is shown in inset of Fig.2.

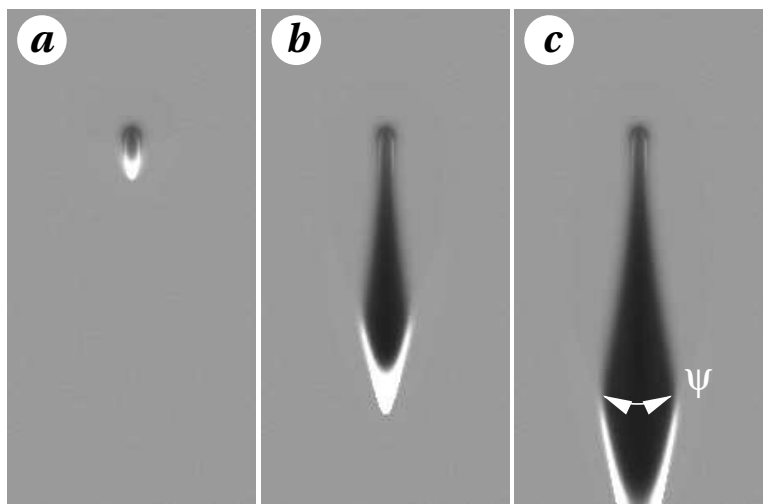

FIG. 3. Grey-coded images demonstrating evolution of triangular avalanche for $t=50$ (a), $t=200$ (b) and 250 (c). White shade correspond to maximum height of the layer, and black to minimum height. Parameters of Eqs. (12,13) are: $\alpha=0.15, \beta=0.25, \delta=1.2$ and $h_{0}=3$, point $A$ in Fig. 2.

For larger values of $\delta$ (close to dashed line in Fig. (2)) or for thicker layers we observed avalanches of the second type. In this case the avalanche propagates also uphill, and contrary to the previous case, the while avalanche zone is in motion, as new rolling particles are constantly arrive from the upper boundary of the avalanche zone. Sometime we observed small secondary avalanches in the wake of large primary avalanche, see Fig. 4tc.
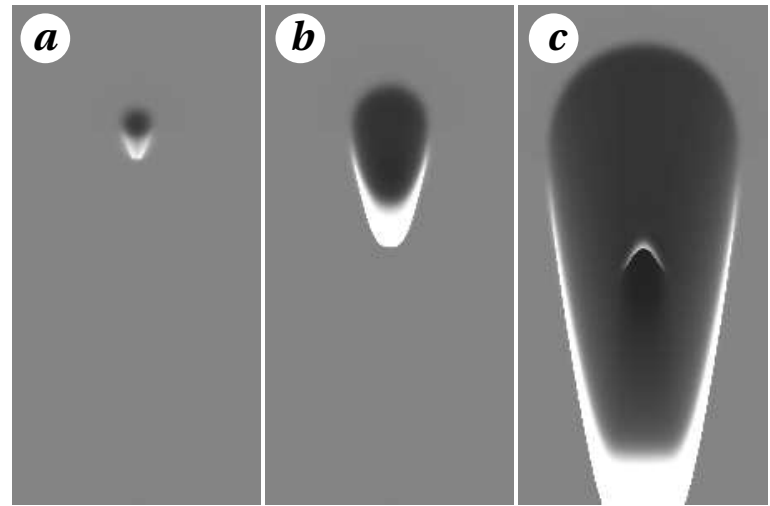

FIG. 4. Images of up-hill avalanche for $t=40$ (a), $t=100$ (b) and 180 (c). Parameters of Eqs. 12, 13) are: $\alpha=0.05, \beta=0.25, \delta=1.07$ and $h_{0}=5.5$, point $B$ in Fig. 2. A small secondary avalanche is seen on the image (c).

In conclusion, we developed a continuum description of partially fluidized granular flows. Our order-parameter model captures important aspects of the phenomenology of chute flows observed in recent experiments [5] 7], including the structure of the stability diagram, triangular shape of downhill avalanches at small inclination angles and balloon shape of uphill avalanches for larger angles. We believe that our model can be applicable to other granular flows including sandpiles and rotating drums.

We thank Dan Howell, Deniz Ertas, Joe Goddard, Bob Behringer and Adrian Daerr for useful discussions. This research is supported by the Office of the Basic Energy Sciences at the US Department of Energy, grants W31-109-ENG-38, DE-FG03-95ER14516, and DE-FG0396ER14592.

[1] H.M. Jaeger, S.R. Nagel, and R.P. Behringer, Physics Today 49, 32 (1996); Rev. Mod. Phys. 68, 1259 (1996)

[2] L. Kadanoff, Rev. Mod. Phys. 71, 435 (1999)

[3] P. G. de Gennes Rev. Mod. Phys. 71, S374 (1999)

[4] R.M. Nedderman, Statics and Kinematics of Granular Materials, (Cambridge University Press, Cambridge, England, 1992)

[5] A. Daerr and S. Douady, Nature (London) 399, 241 (1999)

[6] A. Daerr, unpublished

[7] O. Pouliquen, Phys. Fluids, 11, 542 (1999)

[8] R.A. Bagnold, Proc. Roy. Soc. London A 225, 49 (1954); ibid., 295, 219 (1966)

[9] J. Rajchenbach, in Physics of Dry Granular Media, eds. H. Hermann, J.-P. Hovi, and S. Luding, p. 421, (Kluwer, Dordrecht, 1998); D. McClung, Avalanche Handbook, (Mountaineers, Seattle, 1993)

[10] D. Ertas et al, cond-mat/0005051

[11] O.R. Walton, Mech. Mater. 16, 239 (1993); T. Pöshel, J. Phys. II France 3, 27 (1993); X.M. Zheng and J.M. Hill, Powder Tech. 86, 219 (1996); O. Pouliquen and N. Renaut, J. Phys. II France 6, 923 (1993)

[12] P.G. de Gennes, in Powders \& Grains, R. Behringer \& Jenkins (eds), p.3, Balkema, Rotterdam, 1997

[13] J.-P. Bouchaud et al, J. Phys. I France 4, 1383 (1994)

[14] T. Boutreux, E. Raphaël, and P.G. de Gennes, Phys. Rev. E 58, 4692 (1998)

[15] T. Boutreux and E. Raphaël, Phys. Rev. E 58, 7645 (1998)

[16] L.D.Landau and E.M.Lifshitz, Statistical Physics, Pergamon Press, New York, 1980

[17] S.F. Edwards and R.B.S. Oakeshott, Physica A 157, 1080 (1989); S. F. Edwards and D.V. Grinev, Chaos, 9, $551(1999)$

[18] E. Cantelaube and J.D. Goddard, in Powders \& Grains, R. Behringer \& Jenkins (eds), p.231, Balkema, Rotterdam, 1997; O. Narayan and S.R. Nagel, Physica A 264, 75 (1999).

[19] J.P. Wittmer, M.E. Cates, and P.J. Claudine, J. Phys. II France 7, 39, (1997); L. Vanel et al, Phys. Rev. Lett. 84, 1439 (2000)

[20] M.E. Cates et al, Phys. Rev. Lett. 81, 1841 (1998)

[21] I. S. Aranson, V.A. Kalatsky, and V.M. Vinokur, Phys. Rev. Lett. 84, (2000) 